\title{
TENDENCIAS Y DESAFÍOS DE LA PROGRAMACIÓN MUSICAL*
}

\author{
Miguel Ángel Marín \\ Universidad de La Rioja - Fundación Juan March
}

RESUMEN: Pese a la presencia enraizada que tienen los conciertos de música clásica en nuestra cultura y al auge notable de la gestión musical como disciplina, existe una llamativa ausencia de reflexiones teóricas sobre la programación musical. Este ensayo, redactado desde la perspectiva del programador, se propone hacer una aportación en este ámbito. La primera sección presenta una síntesis histórica del concierto; la segunda esboza un panorama de las principales tendencias de programación musical, particularmente en el ámbito anglosajón; y la tercera analiza los principales desafíos que afronta un programador musical en la actualidad.

Palabras clave: Concierto, programación musical, gestión musical, repertorio, intérprete, público.

\section{TENDENCIES AND CHALLENGES OF MUSICAL PROGRAMMING}

ABSTRACT: Despite the deeply rooted presence of classical music concerts in our culture and the significant growth in music management as a discipline, there is a striking shortage of theoretical reflections on music programming. This article, written from the programmer's point of view, aims to contribute to this field. The first section presents an historical overview of concerts; the second outlines the most important tendencies in music programming, particularly in the Anglo-Saxon

* Agradezco a Luis Martínez su inestimable ayuda en la extracción y gestión de los datos analizados en la segunda sección de este ensayo, y a José Luis Maire la localización de bibliografía especializada, ambos de la Biblioteca de Música y Teatro Contemporáneos de la Fundación Juan March. Este artículo se enmarca en el proyecto I+D HAR2011-22712. 
world; while the third analyses the main challenges a music programmer is faced with today.

Keywords: Concert, music programming, music management, repertory, performer, audience.

Una definición de qué es la programación musical podría resumirse diciendo que es la elección coherente y atractiva de intérpretes y de obras acorde a las posibilidades económicas y al perfil de la institución, así como a los tipos de público destinatarios. Mientras que el primer elemento (el presupuestario) es un condicionante obvio para cualquiera, los dos otros (el perfil institucional y el público destinatario) han suscitado menos reflexión, pese a ser igualmente elementos centrales. Programar es, en esencia, un proceso de toma de decisiones que viene condicionado por cinco variables principales: el repertorio, el intérprete, el presupuesto, el mercado y el público. El resultado final de la programación cristaliza en un acto musical público sobre un escenario. Habría que añadir dos características más propias de la programación: es una actividad que exige una elevada dosis de planificación y tiene un carácter estratégico, en tanto que es un elemento determinante para dotar de una personalidad distintiva a un proyecto o a una institución.

Si asumimos que la programación ocupa, por tanto, un lugar central en la gestión musical, seguramente llamará la atención la poca reflexión teórica que ha suscitado entre los profesionales de este ámbito. Sobre todo si tenemos en cuenta la presencia tan enraizada que tiene el concierto de música clásica como manifestación artística en el panorama actual de la cultura y del ocio. Según la Encuesta de Hábitos y Prácticas culturales en España 2010-2011, elaborada por el Ministerio de Educación, Cultura y Deporte, el 7'7\% de la población española aseguró haber asistido a conciertos de música clásica en el último año. Una década antes, este porcentaje era del 8'4\% (p. 18). Por su parte, el Anuario SGAE de las artes escénicas, musicales y audiovisuales correspondiente al año 2012 indica que el número de conciertos de música clásica en 2011 fue de 15.398 con un total de 4.843.211 asistentes, la cifra más baja de la última década que tuvo su punto culminante en 2006 con 19.399 conciertos a los que asistieron 5.855.205 espectadores (p. 14) 1 . Estos cálculos excluyen otros canales indirectos de difusión de conciertos como la grabación o la transmisión por televisión o radio. Incluso pese al leve descenso de los últimos años, estos datos confirman el impacto notable que tienen los conciertos de música clásica en nuestra sociedad actual.

1. Documentos accesibles en internet: http://www.mcu.es/estadisticas/MC/EHC/2010/ Presentacion.html y http://www.anuariossgae.com/anuario2012/home.html. 
Este ensayo, articulado en tres secciones, está redactado desde la perspectiva de la figura del programador. Comienza con una breve síntesis histórica sobre el concierto público, mencionando los principales cambios que ha sufrido durante los últimos dos siglos y medio aproximadamente, desde su surgimiento hasta el presente. La segunda sección traza un sucinto panorama sobre las tendencias actuales de programación a partir de los datos extraídos de unos 3.000 conciertos celebrados en los últimos cuatro años en distintas ciudades del mundo. Este amplio muestreo desvela los compositores y estilos mejor representados en las salas de conciertos, al tiempo que revela varios rasgos habituales compartidos por programadores y oyentes, aunque quizá no siempre sean del todo conscientes. Por último, el ensayo termina analizando algunos de los principales desafíos que, en mi opinión, afronta actualmente en España un programador de música clásica. Son cuatro los principales frentes con los que este interactúa estableciendo una relación, en ocasiones tácita, de influencia recíproca: el repertorio, el intérprete, el público y los medios de comunicación.

\section{El concierto en perspectiva histórica}

Pese a la revolución tecnológica de las últimas décadas, en el ámbito de la música clásica el concierto sigue siendo la expresión más viva para experimentar este arte. La interpretación de unas obras en un edificio generalmente construido con la función principal de escuchar música (esto y no otra cosa es un concierto) tiene una larga tradición, pues su origen se sitúa a finales del siglo XVIII. El paso de la Edad Moderna a la Contemporánea provocó algunos de los cambios más trascendentales en la historia de la música, cuyas consecuencias siguen siendo todavía hoy plenamente reconocibles. Es una obviedad decir que durante siglos la música fue compuesta para ser interpretada, pero resulta sorprendente afirmar que casi nunca lo fue para ser primordialmente escuchada. La ejecución musical en los espacios habituales hasta entonces perseguía funciones de naturaleza muy diversa: en la iglesia ensalzaba el culto e incitaba a la piedad, en una corte engrandecía la imagen protectora del mecenas, en la calle potenciaba la celebración festiva de una conmemoración y en una casa estimulaba el placer estético y la habilidad interpretativa de sus moradores. Pero solo en el concierto la interpretación de la música tenía como misión principal ser escuchada, en una actitud más o menos atenta, por un público anónimo y variado. En definitiva, el hábito que hoy resulta tan cotidiano de comprar una entrada para acceder a un espacio cerrado con la finalidad principal de escuchar música comenzó aproximadamente hace dos siglos y medio.

Londres y París tuvieron un papel pionero en este proceso, iniciado tímidamente a principios del siglo XVIII. En la mayoría de las ciudades europeas, las primeras temporadas estables de conciertos públicos datan de la segunda mitad de la centuria; en Madrid y en Barcelona, por ejemplo, se fechan en la década 
de $1780^{2}$. Uno de los aspectos más llamativos al comparar estos primeros conciertos con los de hoy es el modo tan distinto que había de programar. En aqueIla época, los conciertos mezclaban géneros y plantillas de un modo que en la actualidad nos resultaría extraño. Durante dos o tres horas, el público escuchaba de ocho a quince obras de géneros diversos como oberturas, sinfonías, arias y conciertos para solista (pero no música de cámara, entonces reservada para los espacios privados, excepto en Londres). Casi todas estas composiciones eran de autores vivos o recientemente fallecidos, incluyendo como norma a varios autores locales. Como veremos a continuación con más detalle, en la actualidad un concierto presenta una media de tres obras y solo excepcionalmente alguna de estas ha sido compuesta en los últimos años.

El surgimiento del concierto público a finales del siglo XVIII corrió en paralelo a la definición del perfil profesional de todos los agentes que participaban en su entramado y lo hacían posible: el empresario u organizador, el representante, el crítico y el articulista, el público y los grupos musicales estables. De hecho, algunas de las orquestas establecidas entonces han sobrevivido hasta el presente. Por ejemplo, la Gewandhaus de Leipzig se fundó en una fecha tan temprana como 1743, la Filarmónica de Viena en 1842, la Filarmónica de Berlín en 1882 y la Orquesta del Concertgebouw de Ámsterdam en 1888.

Conforme avanzó el siglo XIX, el concierto se fue expandiendo y consolidando como un emblema de modernidad social y refinamiento cultural para las ciudades que lo impulsaban. Una de las principales novedades de esta centuria fue la progresiva segmentación de los repertorios y los gustos, que acabaron prefigurando distintos tipos de conciertos en función del público al que se destinaban: concierto de música de cámara, sinfónico, coral, de virtuoso, café-concierto y la gala formada por extractos de ópera y canciones. La práctica compositiva del periodo refleja bien esta transformación social y cultural en tanto que se perfilan con mayor nitidez distintas categorías estéticas y géneros musicales. La música sinfónica, la música de cámara (en particular el cuarteto) y el Lied pasaron a ser considerados géneros elevados que movían el espíritu, frente a otros de carácter ligero como la música de salón o las obras virtuosísticas de lucimiento destinadas al entretenimiento. Cristaliza entonces la definitiva separación -que tiene su origen embrionario mucho antes y se agudizaría durante el siguiente siglo- entre música "seria" y música "popular", por utilizar unas categorías simplificadoras en un proceso complejo, pero comprensibles para cualquier lector.

2. Entre la bibliografía en español, los interesados en la historia del concierto y de la programación encontrarán un buen resumen en William Weber, La gran transformación del gusto musical. La programación de conciertos de Haydn a Brahms, Madrid, Fondo de Cultura Económica, 2012 (original de 2008). Para el caso español, puede verse Miguel Ángel Marín, "Escuchar la música: la academia, el concierto y sus públicos", en José Máximo Leza (ed.), Historia de la Música en España e Hispanoamérica, vol. 4, La música en el siglo XVIII, Madrid, Fondo de Cultura Económica, en prensa. 
En términos de programación, esta transformación culminó hacia finales del siglo XIX y puede resumirse, en palabras de William Weber ${ }^{3}$, en tres cambios fundamentales cuyas consecuencias siguen aún vigentes en la actualidad: 1) el paso de programas misceláneos con géneros diversos a otros homogéneos con menos obras, 2) la práctica desaparición de la alternancia de obras vocales e instrumentales en un mismo concierto, y 3) el abandono de los repertorios contemporáneos del presente en favor de los "clásicos" del pasado. Fue esta fractura la que provocó, con la irrupción de las vanguardias históricas a comienzos del siglo XX, la creación de un nuevo tipo de concierto que persiste en la actualidad, el dedicado exclusivamente a la nueva música. Estos conciertos de música contemporánea (esto es, de cada presente) tenían una vocación rompedora, exclusivista y, en ocasiones, incluso provocadora como nunca antes había ocurrido en la historia de la música. Un ejemplo temprano es la Verein für musikalische Privataufführungen (1919-1921) fundada por Arnold Schöenberg, con conciertos dedicados a obras contemporáneas bien ensayadas, con acceso solo a un público selecto y de los que estaba excluida la prensa, vista por muchos como enemiga del "progreso" musical. Para entonces, el ritual del concierto tal y como lo conocemos hoy llevaba años perfectamente asentado: los carteles publicitarios, los anuncios en la prensa local, la suscripción a la temporada, las notas al programa, la escucha en silencio, los aplausos del público en momentos predefinidos y la sanción de la crítica. Resulta llamativo comprobar la persistencia histórica de estas prácticas, que en su esencia han permanecido inalteradas hasta el presente. Determinar si esta pervivencia se explica por la fórmula de éxito imperecedero que encontró el concierto o por la actitud conservadora y anquilosada de programadores, intérpretes y público merecería una reflexión detenida que trasciende el ámbito de este ensayo ${ }^{4}$.

\section{Tendencias de programación}

Intentar desvelar las tendencias de programación en la música clásica no es una tarea fácil para el caso español. Existen algunos estudios sobre ámbitos muy concretos, la mayoría surgidos en formato de pequeños trabajos de investigación al calor de los diversos másteres de gestión cultural que han aflorado en las últimas dos décadas ${ }^{5}$. Pero no resulta factible, por el momento, tener una

3. Ídem, p. 9.

4. La historia del concierto es un campo de estudio relativamente bien investigado. Pero solo en los últimos tiempos la musicología está interesándose por el concierto como institución social y estética actual. Un ejemplo reciente es Martin Tröndle (ed.), Das Konzert. Neue Aufführungskonzepte für eine klassische Form, Bielefeld, Transcript Verlag, 2009.

5. Dos ejemplos centrados en ámbitos muy determinados que ofrecen estadísticas sobre programación son Ana Guiomar Blanco Souto, El recital de piano en España. Estudio y análisis del repertorio interpretado, Máster de Gestión Cultural: música, teatro y danza, Universidad 
visión de conjunto sobre cómo se programa en España. Desde una óptica internacional es posible tener alguna documentación más amplia. Por ejemplo, en el caso de la ópera, en torno al 75\% de las óperas programadas en una temporada en los principales teatros del mundo pertenecen a solo diez compositores: Mozart, Verdi, Puccini, Wagner, Rossini, Donizetti, Richard Strauss, Bizet, Janacek y Händel. Es más, solo los cuatro primeros autores ya superan el $50 \%$ de las obras programadas ${ }^{6}$.

Cuando este análisis se realiza sobre un grupo más amplio de instituciones musicales, que incluye teatros de ópera pero también auditorios y salas de cámara, el panorama es más variado, pero en el fondo no difiere sustancialmente. La web www.bachtrack.com, fundada en 2008, es una página que reúne los programas de conciertos y óperas celebrados en las principales ciudades del mundo, muchos de los cuales vienen acompañados de recensiones o comentarios. Esta base de datos tiene la finalidad de informar a los aficionados, quienes pueden hacer distintos tipos de búsquedas; incluso ellos mismos, después de validarse ante los responsables, pueden alimentar la base introduciendo programas o recensiones. La riqueza de información y la variedad de instituciones representadas la convierten posiblemente en una base única en su género y permite, al mismo tiempo, vislumbrar algunas tendencias generales de programación. A partir de la información contenida en Bachtrack se ha realizado un muestreo tan amplio como ha sido posible que sirve de soporte para el somero análisis que sigue. El ámbito cronológico del muestro va desde el 1 de enero de 2010 al 15 de julio de 2013 y puede resumirse en las siguiente cifras ${ }^{7}$ :

- Conciertos registrados: 2.770

- Interpretaciones de obras: 8.789

- Instituciones mencionadas: 540

- Ciudades: 132

Una primera conclusión de interés puede extraerse de estos datos con un simple operación matemática. La media de obras interpretadas por concierto es de 3'17, considerablemente por debajo de las entre ocho y quince obras por con-

Complutense, 2005 y Amparo Civera Sáez, La promoción de la música contemporánea desde la Administración Pública. Aproximación al estudio de una política cultural, Máster en Gestión Cultural, Universidad de Valencia, 2010.

6. Según publican Philippe Agid y Jean-Claude Tarondeau, La gestión de la ópera. Un estudio comparativo a nivel internacional, Barcelona, Planeta, 2012, pp. 52-53 (original de 2011). No indican la cronología de la muestra, aunque parecen referirse a una temporada reciente. La información la toman de www.jmb-travel.co.uk.

7. Muestreo realizado a mediados de julio de 2013. Agradezco la generosidad de Bachtrack, en las personas de Alison Karlin y Simon Davies, por haberme permitido utilizar su base de datos para este estudio. 
cierto que era habitual a finales del siglo XVIII. Esta web no persigue fines estadísticos y, por tanto, no tiene voluntad de presentar vaciados sistemáticos de una institución ni datos completos de una ciudad. El muestreo que sirve como base para esta selección muestra, así, dos sesgos que conviene señalar: a) los conciertos analizados son solo los que fueron objeto de una recensión publicada en la propia web, quizá indicador de su mayor atractivo para el público general; y b) la gran mayoría de las 132 ciudades referidas están ubicadas en el Norte de Europa y en Norteamérica, más concretamente en Gran Bretaña, Estados Unidos, Alemania y Canadá. Pero solamente Londres representa un tercio del total de los 2770 conciertos vaciados $\left(36^{\prime} 5 \%\right)$, seguida en la distancia por Nueva York $\left(9^{\prime} 45 \%\right)$. Las siguientes once ciudades de la lista suman el $21^{\prime} 5 \%$, con proporciones que oscilan entre el 3\% y el 1\% (Viena, Sydney, Edimburgo, Mánchester, Montreal, Toronto, Los Ángeles, Ámsterdam, Gateshead, Chicago y Oxford, por este orden). De modo que solo las primeras trece ciudades de la lista acaparan el $66^{\prime} 6 \%$ de las interpretaciones. Si recordamos, además, que ocho de ellas están en Gran Bretaña y Estados Unidos, está claro que las conclusiones que puedan extraerse se aplican de forma prioritaria (aunque no exclusiva) al mundo anglosajón.

Pese a estas limitaciones, el muestreo de conciertos extraído de Bachtrack es suficientemente amplio y variado para poder observar algunas tendencias de programación en el ámbito de la música clásica. El primer rasgo Ilamativo es que durante el periodo seleccionado se han programado obras de 1.265 compositores distintos, una cifra muy considerable que a priori sugiere un rico panorama musical. Sin embargo, un análisis más pormenorizado muestra que esta primera afirmación es problemática. Los autores que han recibido más de cien interpretaciones de las cerca de 8.800 registradas son solo 22. Expresado en términos matemáticos puede decirse que solo el 1'7\% de los autores acaparan nada menos que el $45^{\prime} 7 \%$ del total de interpretaciones. Por orden decreciente, esta lista de oro está conformada por: Beethoven, Mozart, Bach, Brahms, Debussy, Tchaikosvky, Händel, Britten, Schubert, Wagner, Chopin, Schumann, Shostakovich, Stravinsky, Ravel, Richard Strauss, Haydn, Rachmaninov, Liszt, Mahler, Verdi y Prokofiev. Del conjunto de estos autores se realizaron 4.025 interpretaciones de sus obras, que es un número superior a los conciertos registrados (2.770). Se deduce entonces que, como media, en todos estos conciertos al menos uno de ellos estaba representado. La concentración en unos pocos autores es incluso mayor de lo que ya indica este primer análisis, pues solo los diez primeros de la lista (de Beethoven a Wagner en la enumeración anterior) suman casi la cuarta parte de interpretaciones $\left(23^{\prime} 8 \%\right)^{8}$ : en unas 2.100 ocasiones se interpretó alguna obra de esta lista de top ten. Esta cifra equivale al $75 \%$

8. Beethoven: 4\%; Mozart: 3’7\%; Bach: 3'2\%; Brahms: 2'3\%; Debussy 2\%; Tchaikosvky: 1'9\%; Händel: 1'7\%; Britten: 1'7\%; Schubert: 1'7\%; y Wagner: 1'6. Le siguen Chopin y Schumann con el mismo porcentaje. 
de los conciertos del muestreo; es decir, en tres de cada cuatro conciertos se interpretó, como media, una obra de alguno de estos diez compositores.

No es posible determinar qué impacto pudo tener en la configuración de esta lista de autores la coyuntura de los aniversarios. Este tipo de conmemoraciones es un criterio habitual -0 , más bien, una excusa fácil- en la programación, y cabe suponer que no hizo más que intensificar su presencia: Chopin y Schumann en 2010, Mahler en 2011, Debussy y Mahler en 2012, Britten, Verdi y Wagner en 2013. Determinadas tradiciones locales también ayudan a explicar algunas de es-tas tendencias. Por ejemplo, entre los autores más escuchados en Londres, Britten ocupa el puesto número 7 y Carter el 95, mientras que en Nueva York Britten aparece nada menos que en el puesto número 131 y Carter en el 14. De esta comparación se deduce que la presencia destacada de Britten entre los diez compositores más interpretados se explica por la absoluta centralidad que tiene Londres en este muestreo. Si otros países europeos estuvieran mejor representados, es muy posible que el compositor inglés no ocuparía un lugar tan destacado.

Atendiendo al año de fallecimiento de los 22 compositores más interpretados, la mayoría se sitúan en el periodo que transcurre entre comienzos del siglo XIX y la Segunda Guerra Mundial, entre Beethoven (+1827) y Strauss (+1949). Solo seis de ellos se salen de este marco cronológico: Mozart, Bach y Händel son anteriores y Britten, Shostakovich y Stravinsky posteriores (los tres fallecidos en la década de 1970). Esta cronología ya indica con bastante elocuencia dónde se concentra la parte más importante de la programación musical. Pero aún hay más. Los diez compositores más interpretados, que como hemos visto están presentes aproximadamente en el $75 \%$ de los conciertos, murieron hace más de un siglo. El último en fallecer fue Debussy, en $1911^{9}$. La excepción aquí es Britten cuya presencia, como hemos visto, se explica muy probablemente por el enorme peso de los conciertos londinenses en este muestreo. Con estas cifras en mente, la comparación con las tendencias en otras artes, como la literatura, la pintura o el cine, sería un tema para un interesante debate que, sin embargo, trasciende con mucho los objetivos de este ensayo.

En la parte media de la tabla se sitúa un segundo grupo de autores que han recibido entre cien y cuatro interpretaciones. Este grupo intermedio representa aproximadamente un quinto del total de compositores, que están presentes en más de un tercio de los conciertos (véase el cuadro más abajo para los porcentajes precisos). Por último, en el extremo opuesto de la tabla aparecen nada menos que unos mil compositores (el $79^{\prime} 8 \%$ del total) de todas las épocas que

9. Las fechas de fallecimiento son como siguen: Beethoven, 1827; Mozart, 1791; Bach, 1750; Brahms, 1897; Debussy, 1911; Tchaikosvky, 1893; Händel, 1756; Britten, 1976; Schubert, 1827; Wagner, 1888; Chopin, 1849; Schumann, 1857; Shostakovich, 1975; Stravinsky, 1971; Ravel, 1937; Richard Strauss, 1949; Haydn, 1809; Rachmaninov, 1943; Liszt, 1886; Mahler, 1911; Verdi, 1901 y Prokofiev, 1953. 
recibieron tres o menos interpretaciones. De ellos, 720 (56'9\%) solo se interpretaron en una única ocasión. De estas cifras puede deducirse que existe un claro interés por programar a autores muy distintos y por explorar otras estéticas. Pero no tanto como para hacerlo más que de un modo marginal (aunque sea regular en su frecuencia) y sin apenas repetir autores más allá de un club selecto. Dicho en términos prácticos, estas cifras indican que el modus operandi habitual del programador muestra una concentración desmesurada en un grupo muy limitado de compositores, rellenando el concierto con obras de una pléyade de autores diversos poco o nada frecuentes.

El siguiente cuadro resumen la relación entre los compositores representados y el número de interpretaciones que reciben, indicando el porcentaje con respecto a su total:

\begin{tabular}{|l|c|c|}
\hline & Autores & $\begin{array}{c}\text { Frecuencia } \\
\text { (núm. de interpretaciones) }\end{array}$ \\
\hline Más de 100 interpretaciones & $22\left(1^{\prime} 7 \%\right)$ & $4.025(45,74 \%)$ \\
\hline Entre 100 y 4 interpretaciones & $233\left(18^{\prime} 4 \%\right)$ & $3.372(38,32 \%)$ \\
\hline 3 o menos interpretaciones & $1.010\left(79^{\prime} 8 \%\right)$ & $1.392(15,81 \%)$ \\
\hline Total & 1.265 & 8.789 \\
\hline
\end{tabular}

Para el caso español apenas disponemos de datos, y cuando los hay, no siempre permiten una comparación con el panorama descrito para el mundo anglosajón. Un estudio realizado sobre el repertorio interpretado por las 22 orquestas estables existentes en España durante cinco temporadas, entre 1997 y 2002, mostraba incluso una mayor concentración. Los 22 autores más interpretados acaparaban el $49^{\prime} 6 \%$ de las interpretaciones (frente al $40^{\prime} 6 \%$ en el mundo anglosajón). Estos autores son: Mozart, Beethoven, Brahms, Tchaikovsky, Ravel, Bach, Richard Strauss, Stravinsky, Haydn, Mahler, Mendelssonh, Dvorak, Prokofiev, Schubert, Shostakovich, Schumann, Wagner, Bartók, Sibelius, Johann Strauss, Rachmaninov y Bruckner ${ }^{10}$. Resulta inmediata la constatación del sorprendente parecido que esta lista tiene con la anglosajona. Los seis primeros nombres en ambos grupos son iguales, con la diferencia relativa de que Ravel aparece ahora en lugar de Debussy. Solo seis de estos compositores no aparecen entre los 22 más interpretados en los conciertos

10. Estos datos proceden del informe El repertorio de las orquestas sinfónicas en España, Madrid, SGAE y Fundación Autor, 2003, p. 62. 
anglosajones ${ }^{11}$. Los diez primeros de este grupo (de Mozart a Mahler) acaparan el $31^{\prime} 16 \%$ (frente al $23^{\prime} 8 \%$ de los diez primeros en la lista anglosajona).

Como resumen de todos estos datos podemos concluir -con todas las salvedades impuestas por las limitaciones ya apuntados- que la programación en el campo de la música clásica se caracteriza por concentrarse, de un modo bastante acentuado, en dos ámbitos. Por un lado, en una veintena aproximada de compositores que desarrollaron su actividad creativa durante el siglo XIX y principios del siglo XX y, por otro, y como lógica consecuencia, en una estética basada en el repertorio clásico-romántico con incursiones puntuales en las primeras vanguardias. La programación en España, al menos en la música sinfónica, comparte en esencia estas mismas tendencias, con las particularidades propias derivadas de la tradición local, como una presencia proporcionalmente destacada de compositores españoles. Seguramente este panorama no sorprenderá a nadie asiduo a los conciertos. Pero sí ofrece una descripción con base estadística y una primera visión de los detalles más significativos.

\section{Los desafíos de la programación}

La programación se puede entender como la vertiente específicamente artística dentro del campo más amplio de la gestión musical, en el que también se engloban otras tareas de naturaleza administrativa, logística, legal y financiera que quedan fuera del ámbito de este ensayo. Programar implica, en definitiva, promover una negociación explícita o implícita entre los tres agentes que confluyen en la consumación de un concierto: el propio programador, el intérprete y el público. Desde esta perspectiva, el concierto puede también entenderse como el resultado final de una especie de acuerdo o debate a tres bandas entre quienes están en posición para tomar decisiones sobre qué repertorios e intérpretes concurren (el programador), qué obras son factibles de ser preparadas para su ejecución pública (el intérprete) y qué concierto merece la pena o resulta atractivo escuchar en vivo (el público). Este triángulo de negociación sintetiza bien el núcleo de la programación musical.

En buena práctica, los tres agentes deben jugar su papel en el proceso de programación, pues son imprescindibles para la consumación del concierto. Pero no siempre ocurre así. Con demasiada frecuencia, la figura del programador no adquiere la visibilidad que le correspondería por su posición, o solo aparece vinculado a tareas de naturaleza administrativa o presupuestaria. Hay ocasiones en las que podría adoptar un papel activo pero que son relegadas a los intérpretes, como por ejemplo la decisión de qué obras se interpretan o

11. Estos compositores son: Mendelssohn, Dvorak, Bartók, Sibelius, Johann Strauss y Bruckner. La lista anglosajona, donde se contabiliza también la programación de ópera, incluye a Debussy, Händel, Britten, Chopin, Liszt y Verdi. 
cómo se articulan estas dentro del programa. Precisaríamos de un análisis más detallado para aclarar las razones que explican esta praxis. Pero es obvio que deriva en una situación poco deseable, en tanto que supone dejar en manos ajenas decisiones que definen el perfil de la institución. Dicho en términos empresariales, implica desaprovechar una oportunidad estratégica. Esta especie de dejación quizá en parte pueda relacionarse con la escasa tradición de formación de perfiles profesionales específicamente adaptados para la gestión musical, que tengan entre su bagaje un buen conocimiento de la historia de la música y de las herramientas de la musicología. Este escenario no solo empobrece las posibilidades de pensar en programas innovadores, sino que también desvirtúa la interacción de los tres agentes implicados.

En el proceso de negociación artística que implica la organización de un concierto, las posiciones de fuerza entre programador, intérprete y público son asimétricas en tanto que no todos pueden ejercer de igual modo su influencia en la decisión final. Para empezar, la voz del público (o mejor, de los públicos, como veremos enseguida) solo se manifiesta por vía indirecta y, casi siempre, mediante intuiciones a partir de las reacciones que programador e intérprete perciben durante el concierto. Pero además, los intereses de cada uno de estos agentes no son siempre coincidentes en sus detalles, si es que no llegan en ocasiones a ser opuestos. El resto de este ensayo se centra en analizar los que, en mi opinión, son los cuatro retos o desafíos principales que afronta un programador: el repertorio, los intérpretes, el público y los medios de comunicación ${ }^{12}$. Cómo el programador se posiciona ante cada uno de estos desafíos y cómo estos condicionan sus decisiones, es la cuestión de fondo que plantea este ensayo.

\section{El repertorio}

La función esencial del programador es doble. Por un lado, descubrir al oyente (y subsidiariamente también al intérprete) composiciones y autores infrecuentes o poco conocidos que merezcan la pena escucharse en vivo. $\mathrm{Y}$ por otro, proponer nuevos modos de escucha mediante itinerarios novedosos derivados, sobre todo, de una particular articulación del programa o de una puesta en escena poco convencional. Resulta curioso que no se haya reparado en las similitudes evidentes que existen entre un programador musical y un comisario de exposiciones, un perfil profesional bien consolidado en el mercado de la cultura. Ambos comparten el objetivo de proponer formas novedosas o desconocidas de contemplar o escuchar el arte, con recorridos que resulten sorprendentes e inciten a la reflexión, aunque las obras de partida no sean siempre necesaria-

12. Un quinto reto que queda provisionalmente fuera de este análisis es el formato del concierto, que implica aspectos como el lugar de la interpretación, el uso de luces, la colocación del público, la hora y la duración del recital, etc. 
mente inéditas. La elección de las obras, de su disposición en la sala o en el programa, de la perspectiva que presentan y, en definitiva, de la "historia" que cuenta la propuesta son misiones que comparten un programador y un comisario. Como hemos visto, en el caso del concierto la elección queda de facto limitada a un grupo relativamente pequeño de autores y obras, al repertorio canónico de obras musicales compuestas por un número más bien reducido de compositores creadas aproximadamente durante un siglo y medio.

Resulta extraña la persistencia con la que ha pervivido el repertorio clásicoromántico en la programación musical actual. No existen razones estéticas para argumentar su claro predominio sobre el resto de periodos, pero sí hay causas de otra naturaleza. La formación de los intérpretes en los conservatorios está fuertemente basada en estos repertorios, y los rudimentos técnicos de muchos instrumentos todavía se aprenden con los mismos estudios empleados literalmente en el siglo XIX. Pero también es oportuno recordar que, como hemos visto, la propia idea del concierto y el ritual que lo acompaña son frutos del siglo XIX, lo que explica también la íntima vinculación con el repertorio de esa época. Las llamadas música antigua y música contemporánea, hasta hace poco circunscritas a un gueto y todavía secundarias o inexistentes en muchas instituciones musicales, han encontrado sus propios lugares de desarrollo. Es significativo que con mucha frecuencia se hayan acomodado en festivales o ciclos especializados y, por tanto, al margen de las instituciones estables y espacios habituales para la llamada "música clásica". Tanto es así que esta etiqueta tan problemática como arraigada en nuestro vocabulario cotidiano se asocia preponderantemente justo con los periodos Clásico y Romántico de la historia de la música.

En términos históricos, esta situación solo puede entenderse como una anomalía. Durante siglos, la norma fue interpretar casi exclusivamente la música del presente en cualquiera de los espacios donde esta encontró encaje cotidiano: la iglesia, la corte, el salón o, desde finales del siglo XVIII, la sala de conciertos. En primera instancia, los oyentes esperaban encontrar obras de sus contemporáneos escritas en el estilo de su tiempo. Esta práctica sólo comenzó a cambiar, de forma tímida, en la transición del siglo XVIII al XIX cuando se continuó interpretando a algunos compositores, que entonces comenzaron a denominarse "clásicos" después de su muerte. Conforme avanzó el siglo XIX se fue afianzando la noción de que la música del pasado merecería ser rescatada, unas veces como curiosidad erudita y otras como reivindicación de las raíces musicales de un país. El pionero interés de Felix Mendelssohn por recuperar la obra de Bach o, décadas después, el de Ravel por la música de Couperin y Rameau o el de Pedrell y Falla por Victoria, son ejemplos de este lento proceso histórico. Fue solo con la irrupción de las vanguardias a comienzos del siglo XX cuando el divorcio entre el programa de concierto y la música del presente se asentó firmemente. Desde entonces, esta fractura lejos de mitigarse se ha venido acentuando, con un máximo en el periodo de la postguerra. Al tiempo, aumentaba el interés por la música del pasado remoto con la expansión del movimiento de 
la early music, cuyo germen data de finales del siglo XIX pero que solo en los años setenta del pasado siglo eclosionó con fuerza.

Ante este panorama, el primer desafío al que se enfrenta el programador de hoy es la capacidad real que cabe atribuirle y quiera ejercer para, en conjunción con otros agentes como el productor discográfico o los propios intérpretes, alterar esta situación. Es decir, para reconstruir un puente que aminore el divorcio entre público y música del presente. Hay que recordar que esta fractura, por incoherente que resulte en términos históricos y en comparación con otras disciplinas artísticas, en la práctica no parece incomodar ni al público ni a la mayoría de los intérpretes. Lo cual no hace más que intensificar el reto de fondo: la posibilidad de modificar el canon establecido en la sala de conciertos formado, como hemos visto, por una veintena de compositores. No se trata solo de integrar con naturalidad la música contemporánea y la música antigua (entendida como una determinada práctica interpretativa y no como el repertorio de una época concreta) en el repertorio clásico-romántico predominante, sino también de dar más visibilidad a otros autores de este periodo poco o nada representados. En el caso de que tal posibilidad sea factible, la cuestión entonces sería cómo lograr este objetivo, una fórmula que variará según el perfil de las instituciones y los tipos de oyentes, y que pasa irremediablemente por una acción prolongada en el tiempo. El reto es cómo preparar al público para que pueda acercarse y comprender los repertorios contemporáneos, superando la consideración de "cuota de compromiso" con la que con frecuencia operan muchas instituciones musicales. Como en cualquier tipo de elección artística, la selección de las obras, de los intérpretes y -no en último lugar- de la articulación del programa en el que se integran son aspectos determinantes para culminar con éxito una operación de este tipo.

\section{El intérprete}

En buena medida, la posibilidad de innovación de un programador, incentivando obras o compositores que merezcan la pena escucharse en vivo o itinerarios de escucha poco conocidos, está condicionada por la capacidad y la voluntad del intérprete para participar del reto. Pero incluso sin llegar a plantear un escenario innovador, el papel del intérprete es esencial e insustituible en el caso particular de la música, cuya materialización exige irremediablemente el papel de intermediación creativa que encarna el intérprete entre el compositor y el oyente. Es un lugar común recordar algo tan básico como que sin intérprete no hay concierto.

El importante desarrollo de la actividad concertística y de la formación musical que en las últimas décadas ha tenido lugar en Europa, incluyendo también de forma notable a España, ha resultado en la presencia de un elevado número de intérpretes. Este incremento no tendría que equipararse necesariamente con una mayor cualificación artística, aunque en el caso español esta relación parece aceptada por todos, en particular en el ámbito de los solistas (no así todavía 
en el de la música de cámara). La mayor disponibilidad de intérpretes está permitiendo un viraje hacia la contratación de músicos españoles, cada vez más presentes en nuestras temporadas, al tiempo que irremediablemente ha incrementado la competencia entre ellos. Esta circunstancia también ha ocasionado una creciente importancia del marketing y de las estrategias de comunicación. Cada vez más, este tipo de herramientas están ocupando un lugar decisivo en la consolidación de la carrera artística, influyendo de manera subliminal y poderosa -como es el objetivo natural de la mercadotecnia- en la imagen que se proyecta de un artista y que lógicamente también afecta al programador. Hasta tal punto la comunicación y las potentes herramientas que ofrecen las redes sociales están jugando un papel relevante en nuestro mundo globalizado actual en el que el talento de un intérprete -su principal activo- ya no garantiza una carrera de mínima altura. No es incluso imposible que el marketing pueda generar una imagen de valor musical por encima de las capacidades reales del intérprete.

Este panorama cambiante ha traído dos novedades importantes para el programador. Por un lado, una creciente especialización de los intérpretes, que con buen criterio tienden a distinguirse de sus competidores concibiendo proyectos particulares basados en repertorios, épocas, compositores o programas originales. Actúan así como agentes innovadores en la programación. Por otro lado, la presencia más regular de representantes, una figura que funciona como intermediario y negociador entre el intérprete y el programador. El ámbito de actuación de los representantes oscila entre un mero canal de contacto hasta erigirse como el único interlocutor, pasando por distintos estadios intermedios. Su presencia plantea la cuestión a veces espinosa de cómo articular la relación entre un representante y un programador y en qué medida se ven condicionados, en un sentido o en otro, los ideales del programador (o del intérprete).

Como no puede ser de otro modo, el intérprete ejerce un papel central en cualquier intento de "naturalización" -por así decir- en la sala de conciertos de repertorios marginales $u$ olvidados en el sentido explicado anteriormente. No es suficiente con recordar que sin complicidad con un proyecto y sin una vocación genuinamente interesada en abandonar los caminos trillados será difícil que un intérprete logre suscitar el interés del auditorio. Pero este lugar común puede analizarse con más detalle al pensar en cómo una programación juega con lo que podríamos Ilamar el "efecto del estreno". De una parte, el intérprete percibe la posibilidad de ser el primero en interpretar en público una obra o, expresado en términos más crudos, de aprovechar la oportunidad que le surge de presentarse en una sala precisamente porque ha decidido afrontar la preparación del estreno en cuestión. Pero, de otra parte, el esfuerzo para preparar a fondo (como es deseable) una obra desconocida o inédita no siempre podrá amortizarlo en otros conciertos. Se añade que las primeras interpretaciones para el músico, como las primeras audiciones para el público, con frecuencia no son plenamente satisfactorias por la natural falta de rodaje. Y, sin embargo, es inimaginable que tanto el programador como el intérprete renuncien a buscar constantemente nuevos repertorios por descubrir. 


\section{El público}

Entre todos los desafíos que afronta el programador musical, ninguno ha suscitado tanto interés y preocupación como el relacionado con el público. Esto es perfectamente comprensible tan pronto se asume que la mayor o menos asistencia a un concierto tiene dos efectos directos fundamentales: mide el éxito de la propuesta artística y condiciona los ingresos en taquilla. En grados muy distintos según las instituciones, la financiación necesaria para el mantenimiento de la actividad musical y de la propia organización está en relación con su capacidad para generar ingresos por la venta de entradas ( $y$, cada vez más, por otras vías como el mecenazgo).

La cuestión fundamental que siempre planea sobre la cabeza de cualquier programador cuando concibe un proyecto es cómo captar público. La estrategia de comunicación que promueva la institución es un asunto cada vez más determinante, con la irrupción de las redes sociales como un novedoso componente que está revolucionando este campo. Pero es evidente que, en primera instancia, son las características que conforman una programación musical, y no su "venta" entre un público potencial, el elemento esencial para hacerla atractiva. Expresado a la inversa, podría decirse que el público ejerce una influencia determinante aunque a veces inconsciente en los criterios que programadores e intérpretes manejan para seleccionar las obras del concierto. A partir de esta premisa, la primera dificultad estriba en cómo interpretar las preferencias del público de un modo más consistente que la mera intuición. Se puede admitir que la intensidad de los aplausos, los abandonos a mitad del concierto o el índice de ocupación, por citar tres comportamientos perceptibles en una sala, son todos elementos válidos que algo indican sobre el grado de satisfacción del público. Pero es más cuestionable admitir que este tipo de indicadores y otros de naturaleza similar extraídos a través de las reacciones espontáneas in situ permitan obtener una radiografía fiable que guíe con propiedad los criterios de una programación musical.

Quizá sea bueno comenzar asumiendo que en una sala de conciertos no existe un público (en singular) sino varios perfiles de público (en plural): oyentes distintos que reaccionan de modo diferente cuando se enfrentan simultáneamente a la escucha del mismo programa. No sólo pertenecen a diversos estratos sociales y culturales, sino que también tienen expectativas y capacidades de escucha muy distintas. De esta pluralidad no parece deducirse, como se ha tendido a imaginar, una relación directa entre capacidad de escucha y estrato social. El sociólogo y filósofo Theodor W. Adorno, en su conocido ensayo Tipos de comportamiento musical ${ }^{13}$, distinguía nada menos que ocho tipos de oyentes o, para ser más pre-

13. Th. W. Adorno, "Tipos de comportamiento musical", en Introducción a la sociología de la música, Madrid, Akal, 2009, 177-198 (original de 1968), traducción de Gabriel Menéndez Torrellas. Otra clasificación, sin una base sociológica tan fundada y aplicada al caso particular 
cisos, tipos de escucha: experto, buen oyente, consumidor cultural, oyente emocional, oyente resentido, experto en jazz, oyente entretenido y oyente indiferente, no musical y antimusical. No es este el lugar para discutir la validez hoy de esta clasificación concebida en los años sesenta del pasado siglo. Pero al margen de las críticas o revisiones que pudieran plantearse, lo cierto es que el público no es la masa homogénea y compacta que programadores e intérpretes suelen imaginar. Una síntesis de la propuesta adorniana que pueda ser útil como punto de partida preliminar resultaría en los siguientes cinco grupos de oyentes:

1) El experto y el buen oyente con alguna capacitación profesional y capaz de enjuiciar con fundamento una interpretación en términos técnicos y formales;

2) el consumidor de cultura, para quien la música es un bien cultural con valor social, acumulando con algo de fetichismo extensos conocimientos musicales, sobre todo de carácter biográfico y centrados en los intérpretes;

3) el emocional, con una relación menos directa con la música, en la que busca resortes que estimulen y accionen sentimientos y emociones;

4) el resentido, que rechaza la vida musical oficial e institucionalizada, huyendo hacia periodos o repertorios marginados, como podría ser la música antigua o la contemporánea;

5) el entretenido, quizá el perfil predominante, quien carece de una relación específica y crítica con la música, cuya escucha acomete en actitud pasiva y abandonada, sólo como fuente de estímulos sensoriales sin aspirar a desvelar su sentido (cualquier que este pueda ser).

A partir de esta clasificación, el primer desafío que en este terreno afronta un programador es la identificación y proporción de estos perfiles en su sala, algo que difícilmente se podrá establecer a partir de percepciones subjetivas. La configuración de los tipos de oyentes responde a criterios de diversa naturaleza y, entre otros, está condicionada por la propia programación. Y viceversa, pues aquí se da una relación de influencia recíproca entre públicos y programación: los tipos de oyentes de una institución son el resultado de su propia programación y, al mismo tiempo, esta se ajusta según los dictados y preferencias (por lo general, percibidos de un modo indirecto e impreciso) de los perfiles dominantes. En medio de este "fuego cruzado" se mueve el programador, quien está Ilamado a mantener un equilibrio entre la atención a las demandas que cree percibir de sus oyentes y la innovación de una programación que les proporcione el placer del descubrimiento.

de la ópera, puede verse en Herbert Lindenberger, Opera in History. From Monteverdi to Cage, Standord, Standford University Press, 1998, 270-282. 
Una de las dificultades inevitables se plantea cuando las demandas de los oyentes no son coincidentes o entran directamente en conflicto entre sí. Esta circunstancia no puede ser más que predecible intuyendo los distintos intereses musicales de cada perfil. Qué opciones hay de atenderlos a todos de forma equilibrada o qué perfiles se opta por privilegiar son preguntas que no tienen una respuesta universal. Justo en este punto de la argumentación cabría recordar el origen público de una parte importante de la financiación que reciben la práctica totalidad de instituciones musicales en nuestro país. ¿Implica esta circunstancia una cierta obligación moral por parte del programador para atender a los gustos establecidos o, por el contrario, precisamente la financiación pública impone, por así decir, caminar en movimiento contrario para favorecer lo desconocido?

\section{Los medios de comunicación}

La economía de la cultura y de la música, entendidas como bienes de consumo y como espectáculos, está ejerciendo, cada vez más, un papel protagonista en la gestión musical. No es solo que la situación actual esté potenciando la sensibilidad hacia el aspecto financiero que cualquier organización cultural debe cuidar, sino que también es determinante la creciente globalización de la actividad musical. La consolidación de la propia figura del agente musical o el modo en el que hoy se construyen las carreras artísticas son dos de las evidencias más claras, pero también lo son: la existencia de circuitos de intérpretes, la presión mediática que pueden ejercer las discográficas, la voz de la crítica musical, hoy en mínimos históricos en la prensa escrita pero en plena pujanza en los medios digitales más variopintos, los intereses a los que puedan responder las revistas especializadas o las campañas de publicidad, por citar algunos ejemplos.

En este contexto, el programador acaba inevitablemente expuesto al impacto de todo tipo de mensajes surgidos de los medios de comunicación, que de forma consciente o inconsciente moldea su visión sobre el valor de los intérpretes. Cabría plantearse el papel que en la actualidad ejerce la crítica musical y cómo determina, avalando o desechando, las propuestas de programación de una institución. Parece haber consenso en admitir que la fuerza que antaño tuvo la crítica del concierto está ahora en un declive que parece irreversible, o al menos, en una reorientación de su función, cada vez más cercana a la información en detrimento de la evaluación. Pero su voz está siendo sustituida por otro tipo de feed-back menos organizado y en apariencia menos autorizado, pero más influyente y quizá más realista: el que ofrecen oyentes anónimos de a pie en los blogs y en las redes sociales.

Por su parte, la industria discográfica ha jugado durante décadas un papel de importancia creciente que ha corrido paralelo a su expansión como producto de consumo. Incluso en la situación actual, en la que el formato CD está sumido en el camino hacia su definitiva desaparición, sigue siendo percibido por muchos como una herramienta esencial para la construcción de la carrera de un intér- 
prete. La sustitución del CD por otros nuevos formatos digitales, un proceso ahora en marcha, seguramente no eliminará la percepción instalada entre muchos de que la grabación es un elemento imprescindible para la evolución de una carrera musical. Más bien ocurrirá al contrario, en tanto que los nuevos formatos digitales se caracterizan por operar en canales de difusión más rápidos y ágiles. Otra cuestión distinta es en qué medida la proliferación de música grabada, generada en estudio y sometida a un proceso más o menos intenso de posproducción, condiciona y modifica nuestra escucha como oyentes de un concierto en vivo. Llevada esta línea argumental a sus últimas consecuencias, habrá que ver de qué forma el acceso ubicuo e inmediato a grabaciones y la escucha fragmentada, dos rasgos habituales de la música en internet, dejará su huella en nuestros oídos.

En este terreno, el programador aparece situado frente a un entramado de intereses promovidos por los medios de comunicación de naturaleza comercial (aunque no sólo) que le llevan, en cierto sentido, a operar también en claves de mercado. Hasta qué punto puede escapar de los dictados de los medios de comunicación y las expectativas creadas en una parte del público cada vez más dispuesto a pagar solo para escuchar a determinados intérpretes bien atendidos por la crítica y la industria discográfica, es una cuestión digna de reflexión individual. Es seguramente imposible mantenerse por completo ajeno a estas circunstancias, del mismo modo que resulta muy difícil evitar la influencia, a veces inconsciente, de las prácticas y las tendencias actualmente instauradas en las salas de conciertos descritas en este ensayo. Pero ser conscientes de su existencia permite desarrollar una actitud reflexiva en su gestión y canalizar su impacto. Solo podremos modificar aquello que previamente conocemos bien. 